\title{
Analysis of the RDEU Game Model in Mass Emergencies with Maintained Legal Rights by Emotion
}

\author{
Kunpeng Jing and Rui Shi \\ School of Economics and Management, Yanshan University, Qinhuangdao, 066004, China \\ Correspondence should be addressed to Rui Shi; sunson0913@126.com
}

Received 20 June 2018; Revised 11 September 2018; Accepted 30 September 2018; Published 23 October 2018

Academic Editor: Vladimir Turetsky

Copyright (C) 2018 Kunpeng Jing and Rui Shi. This is an open access article distributed under the Creative Commons Attribution License, which permits unrestricted use, distribution, and reproduction in any medium, provided the original work is properly cited.

\begin{abstract}
The emotional factor in mass emergencies with maintained legal rights significantly influences the decision-making of the powerful and vulnerable groups and the course of events. In this paper, we established the game model of rank-dependent expected utility for the powerful and vulnerable groups in mass emergencies with maintained legal rights. And we also explored the influence of different emotional states on the results of strategic equilibrium of both sides of a game. Results show that the behaviours of the powerful or vulnerable groups are not only affected by the choice of their other strategies, but also influenced by the degree of emotion. The more optimistic they are, the more inclined they are to solve the mass emergencies with maintained legal rights; the more pessimistic they are, the more inclined they are to take extreme resistance behaviours and the more likely pessimism is to adversely affect the two groups' behaviour choices. The numerical simulation with MATLAB further validates this conclusion. Therefore, the emotions of both sides should be controlled and understood when managing mass emergencies to solve problems effectively and reasonably.
\end{abstract}

\section{Introduction}

Given the deepening of social transformation from a traditional society to a modern society in China, social contradictions increasingly intensify, especially those manifested by the uneven distribution of benefits among urban and rural areas, social strata, and regions. Thus, all kinds of mass emergencies with maintained legal rights, such as illegal land acquisition and labor disputes, have occurred frequently [1]. According to statistics, about $80 \%$ of domestic mass emergencies are related to rights protection incidents [2]. These incidents greatly affect social stability and harmonious development because they are organized to some extent and featured by intense behavioural unreasonableness. Therefore, the evolution mechanism of these incidents should be explored under the background of a mass emergency with and without maintained legal rights.

Von Neumann et al. assumed that the participants of mass emergencies are "rational persons"; they proposed that the expected utility (EU) theory is a game process where participants seek to maximise their own interests [3]. Lee argued that persons with social backgrounds not only adjusted their behaviour in real time according to the information acquired in the environment, but also change the benefits of people close to them by adjusting their strategies; this finding is not consistent with the assumption of "rational persons" or the fact that decision behaviour ignores irrational factors, such as the cognition and emotions of participants, similar to the Allais paradox [4]. Kahneman et al. [5] attempted to study "bounded rationality" and proposed the prospect theory of nonlinear probability weight functions; they also analysed and explained the strategy-making process of social behaviour and attained considerable achievements. Given the development of neuroscience and technology, some scholars explored the neural mechanism of social behaviour decision-making from deeper levels; they pointed out that the social person displays social preferences, such as trust [6], fairness [7], and altruism [8]. In the game process, personal emotional factors and the reward system of the brain significantly influence behavioural decision-making [9]. Quiggin [10] considered the emotional factors of game participants and proposed the rank-dependent expected utility (RDEU) 
theory to overcome the limitations of EU. Based on the RDEU theory, Xiong and Gong [11, 12] concluded that the state of equilibrium is closely connected with changes in emotion and the scope of games in participants by analysing classic hawk and dove game models and the existence of solutions to the Nash equilibrium. Results showed that emotional factors in the evolution of a mass emergency with maintained legal rights played a very important role in decision-making. Fang [13] considered personal emotion as the key factor that affects strategy and constructed the evolution model of the behaviour of decision makers in mass emergencies. Based on the rank-dependent expected utility theory, Xiong [14] proposed the evolution model of mass emergencies and analysed the effect of emotion on the evolution of events. Chen [15] applied the hypergame analysis method to the cognitive difference in an environmental pollution event by chemical companies; this study also built the hypergame models of both players and combined them with the Dalian Fujia PX event. Based on the rank-dependent expected utility theory, Liu [16] analysed the influence of emotion on the results of a game equilibrium between terrorists and the antiterrorism forces of the government.

Therefore, mass emergencies with maintained legal rights are a typical sudden mass public incident. A substantial number of studies were conducted pertaining to the evolutionary game model in social behaviour decision-making. However, existing studies on behaviour decision-making did not introduce emotional factors to mass emergencies with maintained legal rights and contrasting powers in both sides. This research direction should be developed. Thus, the present study considers the influence of emotional factors on the evolution of a mass public emergency with maintained legal rights. Based on the RDEU theory, the authors constructed a game model of rank-dependent expected utility for powerful and vulnerable groups in mass emergencies with maintained legal rights. This study explored the existence of a solution of different emotional states to the strategic equilibrium of both sides of the game. This approach aims to complement and develop the theoretical methods of evolution of social behaviour decision-making in mass emergencies and provide objective and comprehensive decision-making references.

\section{The RDEU Theory}

The RDEU theory is a nonlinear extension to the linear relation of the probability of the EU theoretical model. The curves are adopted for analysis in the method of a probability triangle. The divergence of an indifference curve is modified and the weight of a nonlinear strategy is established to build the model for the rank-dependent expected utility theory. Many theoretical and empirical studies show that decision weight can be described as the emotional state (optimism or pessimism) and emotional degree of decision makers under uncertain conditions.

Definition 1. If the values of random variable $X$ are from set $\left\{x_{i}, i=1,2, \cdots, n\right\}$, then $x_{i}>x_{2}>\cdots x_{n}$ is obtained and they are subject to probability distribution.

$$
\operatorname{Pr}\left\{X=x_{i}\right\}=p_{i}, \quad i=1,2, \cdots, n
$$

If $p_{i} \geq 0, p_{1}+p_{2}+\cdots+p_{n}=1$ is satisfied, then its ranking position $\left(R P_{i}\right)$ for $x_{i}$ is defined as

$$
\begin{aligned}
R P_{i}=\operatorname{Pr}\left\{X \leq x_{i}\right\}=p_{i}+p_{i+1}+\cdots+p_{n} & \\
& \quad i=1,2, \cdots, n
\end{aligned}
$$

In formula (2), random variable $X$ denotes the benefits that investment decision makers receive by making an investment, which can be intuitively explained by the definition that the higher the ranking position of $x_{i}$, the greater the probability a profit value is not exceeded and the higher the position of $x_{i}$ in the decision-making process.

Definition 2. In risk decision structure $\left\{p_{1}, x_{1} ; p_{2}, x_{2} ; \cdots p_{n}, x_{n}\right\}$, if the utility function of investor is $u(x)$, then the model of rank-dependent expected utility is

$$
V(X, u, \pi)=\sum_{i=1}^{n} \pi\left(x_{i}\right) u\left(x_{i}\right) .
$$

In this formula, $\pi\left(x_{i}\right)$ denotes the decision weight of output $x_{i}$, which can be defined as

$$
\begin{aligned}
& \pi\left(x_{i}\right) \equiv \omega\left(p_{i}+1-R P_{i}\right)-\omega\left(1-R P_{i}\right) \\
& \\
& i=1,2, \cdots, n .
\end{aligned}
$$

In this equation, $\omega(\bullet)$ is a monotone increasing function that satisfies $\omega(0)=0, \omega(1)=1$.

Diecidue and Wakker offer the following propositions according to the definition of (4).

Proposition 3. $\pi\left(x_{i}\right)$ is a monotone decreasing function of ranking position $R P_{i}$ if and only if $\omega$ is a convex function; $\pi\left(x_{i}\right)$ is a monotone increasing function of $R P_{i}$ if and only if $\omega$ is a concave function.

Given cumulative probability $p=p_{1}+p_{2}+\cdots+p_{i}$, that is, the corresponding distribution function $F\left(x_{i}\right)$, then,

$$
\omega\left(p_{i}+1-R P_{i}\right)=\omega\left(F\left(x_{i}\right)\right)=\omega\left(R P_{i}\right) .
$$

Thus, the emotional factor of decision makers can be represented by function $\omega(\bullet)$ in Definition 2. If $\omega(\bullet)$ is a convex function, it means that decision makers have a pessimistic emotion. Decision-making weight $\pi\left(x_{i}\right)$ will tend to decrease as the ranking position of output $x_{i}$ increases. If $\omega(\bullet)$ is a concave function, it means that decision makers have an optimistic emotion. Decision-making weight $\pi\left(x_{i}\right)$ will tend to increase as the ranking position of output $x_{i}$ increases. Therefore, $\omega(\bullet)$ is called an emotional function. We then assume that $\omega_{i}(x)=x^{r_{i}}, r_{i}>0, i=1,2\left(r_{i}\right.$ is the sentiment index of participant $i$ ). This analysis shows that decision makers are considered to have an optimistic emotion when $0<r_{i}<1$. By contrast, decision makers have a pessimistic emotion or they do not have any emotions when $r_{i}=1$. 
TABLE 1: Related parameter hypothesis.

\begin{tabular}{|c|c|}
\hline Parameter & Parameter description \\
\hline$V_{1}$ & $\begin{array}{l}\text { The total income of the powerful group (A), which is obtained by the illegal expropriation of land, } \\
\text { the arrears of wages, and so on }\end{array}$ \\
\hline$V_{2}$ & The total income of the vulnerable group (B) \\
\hline$\beta$ & $\begin{array}{l}\text { Assume that the total strength of the powerful group (A) and the vulnerable group (B) is } 1 \text {. The } \\
\text { strength of the powerful group (A) is } \beta \text {; the vulnerable group (B) is } 1-\beta \text {. So the income of the } \\
\text { powerful group (A) is } \beta V_{1} \text {; the income of the vulnerable group (B) is }(1-\beta) V_{2}\end{array}$ \\
\hline$C_{1}$ & $\begin{array}{c}\text { After an incident, the action costs by the powerful group (A) hardily suppress the vulnerable } \\
\text { group (B) }\end{array}$ \\
\hline$C_{2}$ & $\begin{array}{l}\text { The vulnerable group (B) chooses "struggle," the cost of reputation loss for the powerful group } \\
\text { (A) after seeking media exposure }\end{array}$ \\
\hline$C_{3}$ & $\begin{array}{c}\text { The powerful group (A) commits an illegal act. After the media exposure, the government } \\
\text { intervened to account for the penalty cost }\end{array}$ \\
\hline$C_{4}$ & $\begin{array}{l}\text { The total cost incurred by the vulnerable group (B) in the appeal process and the search for media } \\
\text { help }\end{array}$ \\
\hline
\end{tabular}

TABLE 2: Game payoff matrix for the powerful group and vulnerable group in group public emergency.

\begin{tabular}{lcr}
\hline Vulnerable group $B$ & Tough $\mathrm{T}(p)$ & Powerful group $A$ \\
& $(1-\beta) V_{2}-C_{4}, \beta V_{2}-C_{1}-C_{2}-C_{3}$ & $V_{2}, 0$ \\
Struggle $\mathrm{V}(q)$ & $0, V_{1}$ & $(1-\beta) V_{2}, \beta V_{1}$ \\
Compromised $\mathrm{M}(1-q)$ & & $p)$ \\
\hline
\end{tabular}

Definition 4. If the preference orders " $>$ " of decision makers can be represented by utility function $u(\bullet)$ and weight $\pi(\bullet)$ 's real-valued function $V$, then, the following equation is obtained for random variable $X, Y$ :

$$
\begin{gathered}
X>Y \Longleftrightarrow \\
V(X, u, \pi)>V(Y, u, \pi),
\end{gathered}
$$

where $V$ is the rank-dependent expected utility defined by Model (3); then, the decision makers satisfy the RDEU decision model.

\section{RDEU Game Model for Mass Emergencies with Maintained Legal Rights}

3.1. Basic Game Structure. Mass emergencies occur frequently given rapid economic and social development, accelerated social transformation and widening gap between the rich and poor, and the unbalanced possession of resources and distribution of benefits among sectors of society, which worsen stratum differentiation. Therefore, the powerful group (A), such as enterprise leaders, government departments, and real estate developers, possess more social resources and participate more in the political life of a local society. The powerful group occupies its powerful status because of its members participating degree and influence, whereas the vulnerable group (B), such as grassroots farmers, needy urban workers, and consumers, possess fewer social resources and take a relatively vulnerable position. In mass emergencies with maintained legal rights, the powerful group will adopt two strategies of "cooperation" and "tough fighting," which is denoted as $S_{A}=\{C, T\}$; the probabilities that they will adopt the strategy of "cooperation" and "tough fighting" are denoted as $1-p$ and $p$. Vulnerable groups adopt the strategies of "compromised" and "struggle," which are denoted as $S_{B}=\{M, V\}$, and the probabilities that they will adopt "compromised" and "struggle" are denoted as $1-q$ and $q$.

Related parameter hypothesis is showed in Table 1.

We then assume that the powerful and vulnerable groups are participants with bounded rationality in the rightsdefensive sudden mass incident and that they completely understand the benefit levels, game rules, and game processes of participants. Based on the above assumption, the game revenue matrix for the powerful group and vulnerable group in the mass emergency with maintained legal rights is shown in Table 2.

In parameter hypothesis, the parameter $\beta$ is used to indicate the proportion of the power of the powerful group, $(1-\beta)$ indicates the proportion of the power of the vulnerable group, and the total strength of the powerful group and the disadvantaged group is 1 . According to the actual situation, the strength of powerful political groups such as a company's leadership, real estate development, etc. is stronger than a vulnerable group such as grassroots employees, farmers, and consumers. The powerful groups are obviously stronger than the disadvantaged groups in terms of resource possessions, rights, and information control. Therefore, here $\beta>0.5$; the following will consider the conditions of profit that the two sides of the game have under this condition.

According to this reality, for collective disputes like labor disputes, the dominant group is a corporation or its corporate leadership. They have all the rights to speak about the distribution and dispatch of employees, and they have more social 
TABle 3: Profit value, probability distribution, ranking position, and decision weight of the powerful group in mass emergencies with maintained legal rights.

\begin{tabular}{lccc}
\hline $\begin{array}{l}\text { Income of powerful group } \\
x_{i}\end{array}$ & Probability $p_{i}$ & Ranking position $R P_{i}$ & Decision weight $\pi\left(x_{i}\right)$ \\
\hline$V_{1}$ & $p(1-q)$ & 1 & $\{p-p q\}^{r_{1}}$ \\
$\beta V_{1}$ & $(1-p)(1-q)$ & $1-p+p q$ & $\{1-q\}^{r_{1}}-\{p-p q\}^{r_{1}}$ \\
0 & $q(1-p)$ & $q$ & $\{1-p q\}^{r_{1}}-\{1-q\}^{r_{1}}$ \\
$\beta V_{1}-C_{1}-C_{2}-C_{3}$ & $p q$ & $p q$ & $1-\{1-p q\}^{r_{1}}$ \\
\hline
\end{tabular}

TABle 4: Profit value, probability distribution, ranking position, and decision weight of the vulnerable group in mass emergencies with maintained legal rights.

\begin{tabular}{lccc}
\hline $\begin{array}{l}\text { Income of vulnerable } \\
\text { groups } x_{i}\end{array}$ & Probability $p_{i}$ & Ranking position $R P_{i}$ & Decision weight $\pi\left(x_{i}\right)$ \\
\hline$V_{2}$ & $q(1-p)$ & 1 & $\{q-p q\}^{r_{2}}$ \\
$(1-\beta) V_{2}$ & $(1-p)(1-q)$ & $1-q+p q$ & $\{1-p\}^{r_{2}-\{q-p q\}^{r_{2}}}$ \\
0 & $p(1-q)$ & $p$ & $\{1-p q\}^{r_{2}}-\{1-p\}^{r_{2}}$ \\
$(1-\beta) V_{2}-C_{4}$ & $p q$ & $(1-p)(1-q)$ & $1-\{1-p q\}^{r_{2}}$ \\
\hline
\end{tabular}

resources and source of information channels. As employees of disadvantaged groups, they are the labor force created by an enterprise's value; they have the responsibility of obeying the enterprise and taking the interests of the enterprise as their core interest, with fewer social resources, and in a relatively weak position. From the income $V_{1}>V_{2}$, when the two produce labor disputes, they will choose a struggle strategy to protect their own rights and interests as the vulnerable group. They do not hesitate to pay time and cost to fight or seek the help of the media; these costs are much greater than their income due, $(1-\beta) V_{2}-C_{4}<0$. On the other hand, as a powerful group, to prevent this dispute from expanding, they adopt a tough strategy to suppress the vulnerable groups; in addition to paying suppressed operating costs, they are faced with the loss of reputation after media exposure and after government intervention. The accountability penalty costs are much greater than their income due, that is, $\beta V_{2}-C_{1}-$ $C_{2}-C_{3}<0$. Thus, the income sizes of strong and vulnerable groups with different strategies are as follows:

For powerful groups: $\{$ compromised $1-q$, tough $p\}>$ \{compromised $1-q$, cooperation $1-p\}>$ \{struggle $q$, cooperation $1-p\}>\{$ struggle $q$, tough $p$ \}

For vulnerable groups: $\{$ struggle $q$, cooperation $1-p\}>$ $\{$ compromised $1-q$, cooperation $1-p\}>\{$ compromised $1-$ $q$, tough $p\}>\{$ struggle $q$, tough $p\}$

The parameters meet the following relationship:

$$
\begin{aligned}
& V_{1}>\beta V_{1}>0>\beta V_{1}-C_{1}-C_{2}-C_{3} \\
& V_{2}>(1-\beta) V_{2}>0>(1-\beta) V_{2}-C_{4} .
\end{aligned}
$$

From this, it can be seen that a pure strategy Nash equilibrium exists, \{compromised $1-q$, tough $p$ \} and \{struggle $q$, cooperation $1-p$ \}. When both sides of the game are fully rational, if one side of the decision maker chooses the tough strategy or struggle strategy, the other party of the decision maker will choose the compromise strategy in consideration of maximising their own interests.
In reality, the emotional state of decision makers on both sides of the game is a nonnegligible factor. Therefore, it is necessary to consider that the two sides of the game are not completely rational; that is, we must consider the influence of the emotional state of the decision maker on the choice of game strategy and embody the emotional factors in the utility function.

3.2. Construction of RDEU Game Model for Mass Emergencies with Maintained Legal Rights. The profit value, probability, the corresponding ranking position, and decision weight of the powerful group (A) and vulnerable group (B) based on the RDEU model by Definition 1 are shown in Tables 3 and 4.

The RDEU expected utility function that corresponds to the powerful group is

$$
\begin{aligned}
V_{1}(X, u, \pi)= & \sum_{i=1}^{n} \pi\left(x_{i}\right) u\left(x_{i}\right) \\
= & V_{1} \cdot(p-p q)^{r_{1}}+\beta V_{1} \\
& \cdot\left\{(1-q)^{r_{1}}-(p-p q)^{r_{1}}\right\}+0 \\
& +\left(\beta V_{1}-C_{1}-C_{2}-C_{3}\right) \\
& \cdot\left\{1-(1-p q)^{r_{1}}\right\} .
\end{aligned}
$$

The RDEU expected utility function that corresponds to the vulnerable group is

$$
\begin{aligned}
V_{2}(X, u, \pi)= & \sum_{i=1}^{n} \pi\left(x_{i}\right) u\left(x_{i}\right) \\
= & V_{2} \cdot(q-p q)^{r_{2}}+\left(1-\beta V_{2}\right) \\
& \bullet\left\{(1-p)^{r_{2}}-(q-p q)^{r_{2}}\right\}+0 \\
& +\left\{(1-\beta) V_{2}-C_{4}\right\} \cdot\left\{1-(1-p q)^{r_{2}}\right\} .
\end{aligned}
$$


3.3. Analysis of Equilibrium Solution of RDEU Game Model for Mass Emergencies with Maintained Legal Rights. We need to determine the partial derivative by using the expected utility function of (8) and (9) for $p$ and $q$ and compose the partial derivative results into partial equations as (12).

$$
\begin{aligned}
& \frac{\partial V_{1}(p, q)}{\partial p}=r_{1}\left\{(1-\beta)(1-q) V_{1}(p-p q)^{r_{1}-1}\right. \\
& \left.\quad+q\left(\beta V_{1}-C_{1}-C_{2}-C_{3}\right)(1-p q)^{r_{1}-1}\right\} \\
& \frac{\partial V_{2}(p, q)}{\partial q}=r_{2}\left\{\beta(1-p) V_{2}(q-p q)^{r_{2}-1}\right. \\
& \left.\quad+p\left[(1-\beta) V_{2}-C_{4}\right](1-p q)^{r_{2}-1}\right\} \\
& \frac{\partial V_{1}(p, q)}{\partial p}=r_{1}\left\{(1-\beta)(1-q) V_{1}(p-p q)^{r_{1}-1}\right. \\
& \left.\quad+q\left(\beta V_{1}-C_{1}-C_{2}-C_{3}\right)(1-p q)^{r_{1}-1}\right\} \\
& \frac{\partial V_{2}(p, q)}{\partial q}=r_{2}\left\{\beta(1-p) V_{2}(q-p q)^{r_{2}-1}\right. \\
& \left.\quad+p\left[(1-\beta) V_{2}-C_{4}\right](1-p q)^{r_{2}-1}\right\} .
\end{aligned}
$$

In (12), both equations are 0 at the same time. Conditions for the Nash equilibrium solution of the RDEU game model for the strategy of powerful and vulnerable groups in the rights-defensive sudden mass incident are obtained as follows:

$$
\begin{aligned}
& (1-\beta)(1-q) V_{1}(p-p q)^{r_{1}-1} \\
& \quad+q\left(\beta V_{1}-C_{1}-C_{2}-C_{3}\right)(1-p q)^{r_{1}-1}=0 \\
& \beta(1-p) V_{2}(q-p q)^{r_{2}-1} \\
& \quad+p\left[(1-\beta) V_{2}-C_{4}\right](1-p q)^{r_{2}-1}=0 .
\end{aligned}
$$

Equation (13) is a transcendental equation with an analytical solution that is difficult to obtain. Therefore, the existence of a solution to the equation is discussed in the following three typical situations.

Situation 5 (when both sides are under a rational state). In the game, both the vulnerable group and powerful group are under a rational status, that is, $r_{1}=1, r_{2}=1$. The following equation is obtained by substituting it into (13):

$$
\begin{aligned}
(1-\beta) V_{1}(1-q)+\left(\beta V_{1}-C_{1}-C_{2}-C_{3}\right) q & =0 \\
\beta V_{2}(1-p)+\left[(1-\beta) V_{2}-C_{4}\right] p & =0 .
\end{aligned}
$$

The powerful group adopts a mixed strategy as follows:

$$
\begin{aligned}
& (p, 1-p) \\
& \quad=\left(\frac{\beta V_{2}}{(2 \beta-1) V_{2}+C_{4}}, 1-\frac{\beta V_{2}}{(2 \beta-1) V_{2}+C_{4}}\right) .
\end{aligned}
$$

The vulnerable group also adopts a mixed strategy:

$$
\begin{gathered}
(q, 1-q)=\left(\frac{(1-\beta) V_{1}}{(1-2 \beta) V_{1}+C_{1}+C_{2}+C_{3}}, 1\right. \\
\left.-\frac{(1-\beta) V_{1}}{(1-2 \beta) V_{1}+C_{1}+C_{2}+C_{3}}\right) .
\end{gathered}
$$

When $r_{1}=1, r_{2}=1$, the reaction functions of the powerful groups and vulnerable groups are

$$
\begin{aligned}
& p= \begin{cases}1 & \text { if } q<\frac{(1-\beta) V_{1}}{(1-2 \beta) V_{1}+C_{1}+C_{2}+C_{3}} \\
{[0,1]} & \text { if } q=\frac{(1-\beta) V_{1}}{(1-2 \beta) V_{1}+C_{1}+C_{2}+C_{3}} \\
0 & \text { if } q>\frac{(1-\beta) V_{1}}{(1-2 \beta) V_{1}+C_{1}+C_{2}+C_{3}}\end{cases} \\
& q= \begin{cases}1 & \text { if } p<\frac{\beta V_{2}}{(2 \beta-1) V_{2}+C_{4}} \\
0,1] & \text { if } p=\frac{\beta V_{2}}{(2 \beta-1) V_{2}+C_{4}} \\
0 & \text { if } p>\frac{\beta V_{2}}{(2 \beta-1) V_{2}+C_{4}} .\end{cases}
\end{aligned}
$$

The Nash equilibrium of mixed strategy is

$$
\begin{aligned}
& \left(p^{*}, q^{*}\right) \\
& =\left(\frac{\beta V_{2}}{(2 \beta-1) V_{2}+C_{4}}, \frac{(1-\beta) V_{1}}{(1-2 \beta) V_{1}+C_{1}+C_{2}+C_{3}}\right) .
\end{aligned}
$$

The situations wherein both sides of the game have no emotions become special circumstances that are identical with traditional game theory, but these are not common in real life.

Situation 6 (at least one side has an emotional state). In real life, the powerful groups that have more social resources are in a socially advantageous position. However, vulnerable groups have a small number of social resources placing them in a disadvantageous position. When information asymmetry exists, the vulnerable groups are, in most cases, emotional. Emotion will largely affect their decision-making. They will then be likely to adopt extreme methods to defend their rights. Therefore, we assume that, for the powerful group, the government and other administrators are rational persons without emotions, whereas the vulnerable groups have emotions. Based on the characteristics of emotional functions in the RDEU game model of group conflict, the emotions of vulnerable groups can be divided into an "optimistic emotion" and a "pessimistic emotion."

Hypothesis 7. We then assume that the vulnerable groups hold an optimistic attitude towards the future and the powerful group has a rational attitude. The sentiment index of vulnerable groups is $r_{2}>1$; that is, the emotional function $\omega_{2}(p)$ is a convex function, whereas the emotional function of the powerful group is $r_{1}=1$ and we assume there is no 
emotion. In real life, vulnerable groups have a high degree of trust in the government and they seek help from media, from the Internet, through petition letters, or from government departments. These approaches provide conditions favorable to their rights-defensive behaviour. Thus, they hold an optimistic attitude towards the game result.

When $r_{2} \longrightarrow+\infty$, that is, the optimistic emotion of vulnerable groups turns from the bounded rationality to nonrationality, then $r_{2}-1 \longrightarrow+\infty$. Equation (13) shows that

$$
\begin{aligned}
& \beta(1-p) V_{2}(q-p q)^{r_{2}-1} \\
& \quad+p\left[(1-\beta) V_{2}-C_{4}\right](1-p q)^{r_{2}-1}=0 .
\end{aligned}
$$

Then, $((q-p q) /(1-p q))^{r_{2}-1}=\left[C_{4}-(1-\beta) V_{2}\right] p / \beta V_{2}(1-p)$.

Given that $q-p q<1-p q$, then $(q-p q) /(1-p q)<1$. Therefore, $((q-p q) /(1-p q))^{r_{2}-1} \longrightarrow 0$, that is, $p \longrightarrow 0$, and the mixed strategy that the vulnerable groups adopt is

$$
\begin{gathered}
(q, 1-q)=\left(\frac{(1-\beta) V_{1}}{(1-2 \beta) V_{1}+C_{1}+C_{2}+C_{3}}, 1\right. \\
\left.-\frac{(1-\beta) V_{1}}{(1-2 \beta) V_{1}+C_{1}+C_{2}+C_{3}}\right) .
\end{gathered}
$$

The strategy probability that the powerful group adopts is $(p, 1-p)=(0,1)$. Therefore, the Nash equilibrium of mixed strategy is

$$
\left(p^{*}, q^{*}\right)=\left(0, \frac{(1-\beta) V_{1}}{(1-2 \beta) V_{1}+C_{1}+C_{2}+C_{3}}\right) .
$$

Hypothesis 8. Vulnerable groups have pessimistic attitudes and the behaviour of powerful groups is rational. The sentiment index of vulnerable groups is $0<r_{2}<1$; that is, the emotional function $\omega_{2}(p)$ is a concave function. The sentiment index of powerful groups is $r_{1}=1$, which is rational. In real life, the basic-level government has low prestige among people and the vulnerable groups have a relatively low degree of trust in the government. They believe that the only way to spread mass emergencies with maintained legal rights is by means of "struggle." Thus, they hold pessimistic attitudes reflected in the game results.

If $r_{2} \longrightarrow 0$, that is, the emotional state of a vulnerable group gradually becomes pessimistic, then $r_{2}-1 \longrightarrow-1$ as shown by (13):

$$
\begin{aligned}
& \beta(1-p) V_{2}(q-p q)^{r_{2}-1} \\
& \quad+p\left[(1-\beta) V_{2}-C_{4}\right](1-p q)^{r_{2}-1}=0 .
\end{aligned}
$$

Then, $((p-p q) /(p+q-p q))^{r_{2}-1}=(h /(e-f))((1-p) / p)$. as

The mixed strategy vulnerable groups adopt is then given

$$
\begin{gathered}
(q, 1-q)=\left(\frac{(1-\beta) V_{1}}{(1-2 \beta) V_{1}+C_{1}+C_{2}+C_{3}}, 1\right. \\
\left.-\frac{(1-\beta) V_{1}}{(1-2 \beta) V_{1}+C_{1}+C_{2}+C_{3}}\right)
\end{gathered}
$$

$$
\begin{aligned}
& =\left(\frac{(1-\beta) V_{1}}{(1-2 \beta) V_{1}+C_{1}+C_{2}+C_{3}},\right. \\
& \left.\frac{-\beta V_{1}++C_{1}+C_{2}+C_{3}}{(1-2 \beta) V_{1}+C_{1}+C_{2}+C_{3}}\right) .
\end{aligned}
$$

The mixed strategy powerful groups adopt is then given as

$$
\begin{aligned}
& (p, 1-p)=\left(\frac{\beta\left[(1-2 \beta) V_{1}+C_{1}+C_{2}+C_{3}\right]}{(1-\beta)\left[(1+2 \beta) V_{2}+C_{4}\right]}, 1\right. \\
& \left.\quad-\frac{\beta\left[(1-2 \beta) V_{1}+C_{1}+C_{2}+C_{3}\right]}{(1-\beta)\left[(1+2 \beta) V_{2}+C_{4}\right]}\right) .
\end{aligned}
$$

The Nash equilibrium of this mixed strategy is

$$
\begin{aligned}
& \left(p^{*}, q^{*}\right)=\left(\frac{\beta\left[(1-2 \beta) V_{1}+C_{1}+C_{2}+C_{3}\right]}{(1-\beta)\left[(1+2 \beta) V_{2}+C_{4}\right]},\right. \\
& \left.\frac{(1-\beta) V_{1}}{(1-2 \beta) V_{1}+C_{1}+C_{2}+C_{3}}\right)
\end{aligned}
$$

In real life, when group conflict reaches a certain level, the vulnerable groups will fight fiercely because their own interest is hampered and affected by their own emotions. They will then perform aggressive actions. Offensive behaviour manifests, such as bloody violence, and the situation worsens. However, the powerful side that occupies the dominant position will take actions based on their own advantage to defend their own benefits. Thus, mass emergencies may occur. Therefore, in a game with powerful groups and vulnerable groups, they should adjust their unreasonable strategies and avoid the occurrence of mass emergencies. The government and relevant administrative departments should also improve the coordination and arbitration mechanism for these mass emergencies.

Situation 9 (both sides have emotion). In this situation, $r_{1} \neq$ $1, r_{2} \neq 1$, the reaction functions of the powerful group and vulnerable groups are

$$
\begin{aligned}
\frac{(1-q)(1-\beta) V_{1}}{q\left(C_{1}+C_{2}+C_{3}-\beta V_{1}\right)} & =\left(\frac{1-p q}{p-p q}\right)^{r_{1}-1} \\
\frac{(1-p) \beta V_{2}}{p\left(C_{4}-(1-\beta) V_{2}\right)} & =\left(\frac{1-p q}{q-p q}\right)^{r_{2}-1} .
\end{aligned}
$$




$$
\begin{aligned}
& p= \begin{cases}1 & \text { if } q=0 \\
\left\{\left[\left(\frac{(1-\beta) V_{1}}{C_{1}+C_{2}+C_{3}-\beta V_{1}}\right)\left(\frac{1}{q}-1\right)\right]^{1 /\left(r_{1}-1\right)}(1-q)+q\right\}^{-1} & \text { if } q \in(0,1)\end{cases} \\
& q= \begin{cases}1 & \text { if } q=1 . \\
\left\{\left[\left(\frac{\beta V_{2}}{C_{4}-(1-\beta) V_{2}}\right)\left(\frac{1}{p}-1\right)\right]^{1 /\left(r_{2}-1\right)}(1-p)+p\right\}^{-1} & \text { if } p=0 \\
0 & \text { if } q \in(0,1)\end{cases} \\
&
\end{aligned}
$$

To examine the influence of changes in sentiment index $r_{1}, r_{2}$ on the decision-making of gamers in (27) and (28), consider $p, q$ as the functions of $r_{1}, r_{2}$. Therefore, $p$ is subject to the influence of $r_{1}$, denoted as $p\left(r_{1}\right) ; q$ is subject to the influence of $r_{2}$, denoted as $q\left(r_{2}\right)$. The first-order derivative for $p\left(r_{1}\right)$ and $p\left(r_{2}\right)$, as shown in (29) and (30), is solved by analysing the changes in the curve $p, q$. Accordingly, the influence of $r_{1}, r_{2}$ on $p, q$ is determined, and $p^{\prime}\left(r_{1}\right)$ and $q^{\prime}\left(r_{2}\right)$ are given as

$$
\begin{aligned}
& p^{\prime}\left(r_{1}\right) \\
& =\frac{(1-q) \ln \left[\left((1-\beta) V_{1} /\left(C_{1}+C_{2}+C_{3}-\beta V_{1}\right)\right)(1 / q-1)\right]\left[\left((1-\beta) V_{1} /\left(C_{1}+C_{2}+C_{3}-\beta V_{1}\right)\right)(1 / q-1)\right]^{1 /\left(r_{1}-1\right)}}{\left(r_{1}-1\right)^{2}\left\{q+(1-q)\left[\left((1-\beta) V_{1} /\left(C_{1}+C_{2}+C_{3}-\beta V_{1}\right)\right)(1 / q-1)\right]^{1 /\left(r_{1}-1\right)}\right\}^{2}} \\
& q^{\prime}\left(r_{2}\right)=\frac{(1-p) \ln \left[\left(\beta V_{2} /\left(C_{4}-(1-\beta) V_{2}\right)\right)(1 / p-1)\right]\left[\left(\beta V_{2} /\left(C_{4}-(1-\beta) V_{2}\right)\right)(1 / p-1)\right]^{1 /\left(r_{2}-1\right)}}{\left(r_{2}-1\right)^{2}\left\{p+(1-p)\left[\left(\beta V_{2} /\left(C_{4}-(1-\beta) V_{2}\right)\right)(1 / p-1)\right]^{1 /\left(r_{2}-1\right)}\right\}^{2}} .
\end{aligned}
$$

Equation (29) shows that the denominator is constantly larger than zero. Thus, the plus or minus of $p^{\prime}\left(r_{1}\right)$ in intervals $(0,1)$ and $(1,+\infty)$ depends on the plus or minus of the numerator, that is, the plus or minus of $\ln \left[\left((1-\beta) V_{1} /\left(C_{1}+\right.\right.\right.$ $\left.\left.\left.C_{2}+C_{3}-\beta V_{1}\right)\right)(1 / q-1)\right]$, which determines whether or not $\left((1-\beta) V_{1} /\left(C_{1}+C_{2}+C_{3}-\beta V_{1}\right)\right)(1 / q-1)$ is greater than 1 . In other words, it is necessary to determine the sizes of $q$ and $(1-\beta) V_{1} /\left((1-2 \beta) V_{1}+C_{1}+C_{2}+C_{3}\right)$. For convenience, let $(1-\beta) V_{1} /\left((1-2 \beta) V_{1}+C_{1}+C_{2}+C_{3}\right)=A$.

(1) When $q=A, p^{\prime}\left(r_{1}\right)=0$, and $p\left(r_{1}\right)$ does not change as the sentiment index $r_{1}$ changes. The strategy of a vulnerable group will affect the strategy of the powerful group at the critical value; the powerful group will determine the corresponding strategy.

(2) When $q<A, p^{\prime}\left(r_{1}\right)>0$, the function $p\left(r_{1}\right)$ is an increasing function about $r_{1}$; that is, the probability that the vulnerable group adopts the "struggle" strategy is smaller than the critical value A and is getting smaller and smaller. As the powerful group becomes more and more optimistic, more and more believe that the vulnerable group will compromise. In order to reduce the cost, the probability that the powerful group chooses "tough" strategy $p\left(r_{1}\right)$ increases, so the equilibrium of the game tends to be (compromised, tough).
(3) When $q>A, p^{\prime}\left(r_{1}\right)<0$. The function $p\left(r_{1}\right)$ is a decreasing function about $r_{1}$. With the increase of $r_{1}$, the powerful group is getting more and more pessimistic. They believed that the vulnerable group will not solve the problem peacefully. In order to reduce the cost, the probability that the powerful group chose "tough" increases, so the equilibrium of the game tends to be (struggle, tough).

Three results are obtained after analysing (30).

Equation (30) shows that the denominator is constantly larger than zero. Thus, the plus or minus of $q\left(r_{2}\right)$ in intervals $(0,1)$ and $(1,+\infty)$ depends on the plus or minus of the numerator, that is, the plus or minus of $\ln \left[\left(\beta V_{2} /\left(C_{4}-(1-\right.\right.\right.$ $\left.\left.\left.\beta) V_{2}\right)\right)(1 / p-1)\right]$, which determines whether or not $\left(\beta V_{2} /\left(C_{4}-\right.\right.$ $\left.\left.(1-\beta) V_{2}\right)\right)(1 / p-1)$ is greater than 1 . In other words, it is necessary to determine the sizes of $p$ and $\beta V_{2} /\left((2 \beta-1) V_{2}+\right.$ $\left.C_{4}\right)$. For convenience, let $\beta V_{2} /\left((2 \beta-1) V_{2}+C_{4}\right)=B$.

(4) When $p=B$ and $q^{\prime}\left(r_{2}\right)=0, q\left(r_{2}\right)$ does not change as sentiment index $r_{2}$ changes. The strategy of powerful groups will affect the strategy of vulnerable groups at the critical value and the vulnerable groups will determine the corresponding strategy.

(5) When $p<B$ and $q^{\prime}\left(r_{2}\right)>0$, function $q\left(r_{2}\right)$ is an increasing function about $r_{2}$, and it increases as $r_{2}$ increases. The probability that the vulnerable group adopts the "struggle" strategy is smaller than the critical value B 


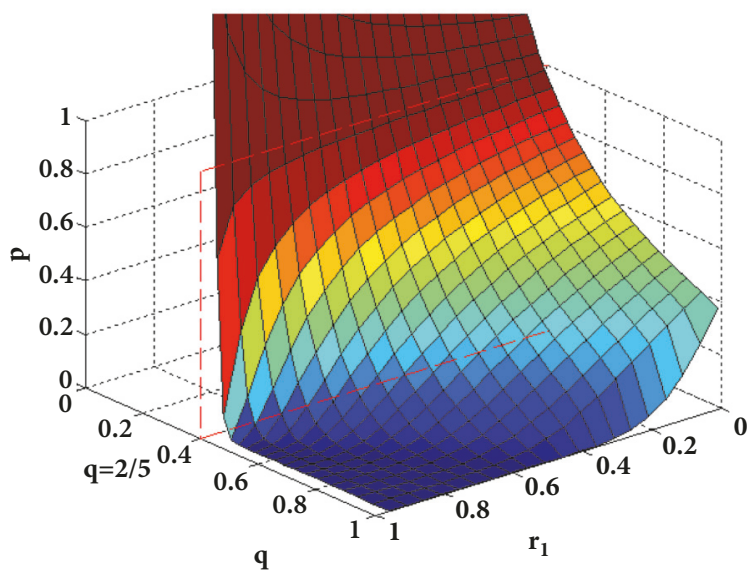

(a) When $r_{1} \in(0,1)$, the powerful group will adopt "tough fighting" strategy $p$ subject to the changes in the pessimistic emotion $r_{1}$ and $q$ influence under a three-dimensional perspective. The deeper the blue color, the smaller the $p$ value, the larger the $q$ value, and the larger the $r_{1}$ value

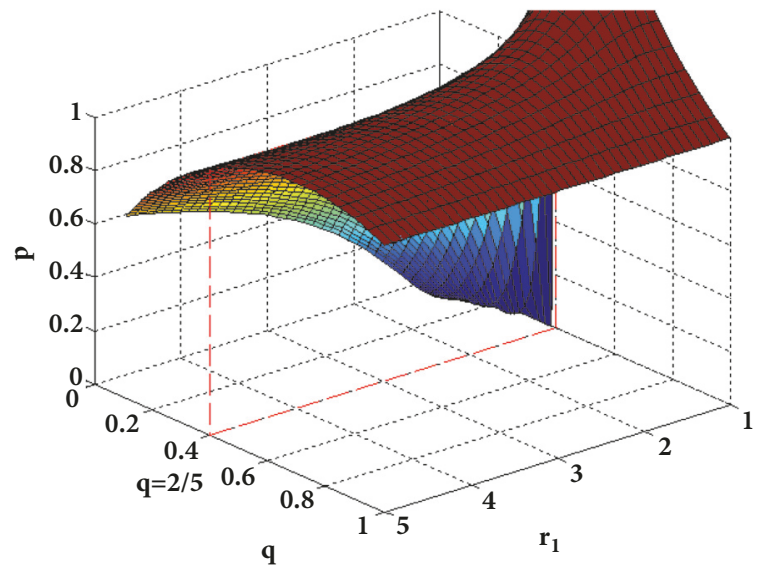

(b) When $r_{1} \in(1,5)$, the powerful group will adopt "tough fighting" strategy $p$ subject to the changes in the optimistic emotion $r_{1}$ and $q$ influence under a three-dimensional perspective. The deeper the blue color, the smaller the $p$ value, the smaller the $q$ value, and the smaller the $r_{1}$ value and is getting smaller and smaller. The probability $q\left(r_{2}\right)$ that the group will choose the strategy of "cooperation" will increase accordingly. As the powerful group becomes more and more optimistic, more and more believe vulnerable groups will certainly compromise. In order to reduce the cost, the probability that the powerful group chooses "tough" strategies $p\left(r_{1}\right)$ will increase, so the equilibrium of the game tends to be (struggle, cooperation).

(6) When $p>B$ and $q^{\prime}\left(r_{2}\right)<0$, function $q\left(r_{2}\right)$ is a decreasing function of $r_{2}$, which decreases as $r_{2}$ increases. When the probability that the powerful group selects a "strategy" strategy is greater than the critical value $B$, the probability that the powerful group chooses the "cooperation" strategy is decreasing. Then the vulnerable group is getting more and more pessimistic; they will become increasingly pessimistic and increasingly distrustful. In order to reduce losses, the probability of their "struggle" choice strategy increases, so the equilibrium of the game tends to be (struggle, tough).

\section{Numerical Analysis}

To better observe the process that the strategy of one side of the game affects the strategy of the other side and their own emotion, the "Jinning incident" in 2014 [17] is selected as a typical case for a mass emergency with maintained legal rights. In this incident, the local villagers have not been able to solve the problem of compensation with the project's constructor about the land acquisition. So we assume that the project constructor is the powerful group (A) and the villagers are the vulnerable group (B). Obviously, the strength of the powerful group is greater than the vulnerable group. Thus, we assume the strength of the powerful group is three times that of the vulnerable group, that is, $\beta=0.75$. The developers promised $¥ 180$ per square meter of compensation initially has become $¥ 43$ thousand per person. While the average per capita income of the local villagers is about $¥ 30$ thousand per year, each family has at least three or four persons. According to the local government's compensation policy of 1:1.3, the values of each factor in the game matrix are roughly assumed as $V_{1}=8, V_{2}=6, C_{1}=4, C_{2}=3, C_{3}=$ 2, $\mathrm{C}_{4}=5$.

According to this assumption, the corresponding critical value is

$$
\begin{aligned}
& p=\frac{\beta V_{2}}{(2 \beta-1) V_{2}+C_{4}}=\frac{9}{16}, \\
& q=\frac{(1-\beta) V_{1}}{(1-2 \beta) V_{1}+C_{1}+C_{2}+C_{3}}=\frac{2}{5} .
\end{aligned}
$$

The changes in MATLAB simulated sentiment index $r_{1}, r_{2}$ are used to reflect the process that the emotions affect the strategies of both sides of a game as shown in Figure 1 because both the powerful group and vulnerable groups have emotions.

(1) From vulnerable groups

Figure 1(a) $r_{1} \in(0,1)$ shows that the powerful group has a "pessimistic emotion" when $p<2 / 5$; the probability that the powerful group adopts the "tough" strategy $p$ increases as $r_{1}$ increases. The smaller $q$ is, the higher $p$ will be. By contrast, when $p>2 / 5$, the probability that the powerful group adopts the "tough" strategy $p$ decreases as $r_{1}$ increases. The greater $q$ is, the lower $p$ will be.

Figure 1(b) $r_{1} \in(1,5)$ shows the powerful group has an "optimistic emotion," which is similar to the case of $r_{1} \in(0,1)$. The results of the two figures show that the increase and decrease of $r_{1}$ for the "pessimistic emotion" in $r_{1} \in(0,1)$ and "optimistic emotion" in $r_{1} \in(1,5)$ have different influences on the probability $q$. The "pessimistic emotion" shows that the vulnerable group will have stronger influence than the "optimistic emotion" on decision-making, 


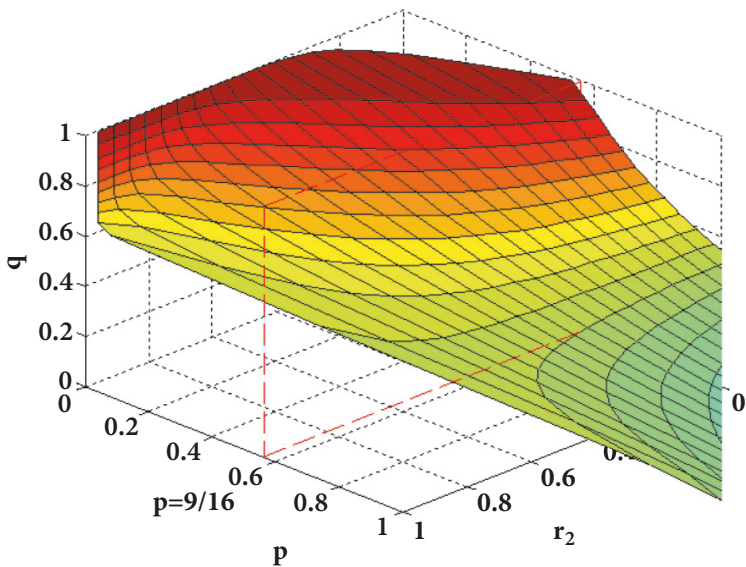

(a) When $r_{2} \in(0,1)$, the vulnerable group will adopt "struggle" strategy $q$ subject to the changes in the pessimistic emotion $r_{2}$ and $p$ influence under a three-dimensional perspective. The deeper the green color, the smaller the $q$ value, the larger the $p$ value, and the smaller the $r_{2}$ value

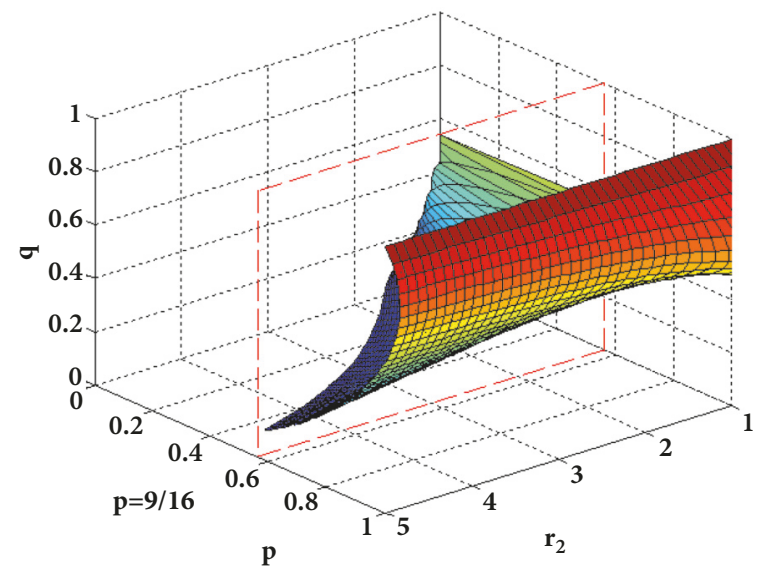

(b) When $r_{2} \in(1,5)$, the vulnerable group will adopt "struggle" strategy $q$ subject to the changes in the optimistic emotion $r_{2}$ and $p$ influence under a three-dimensional perspective. The deeper the blue color, the smaller the $q$ value, the smaller the $p$ value, and the larger the $r_{2}$ value

Figure 2

whereas the pessimistic emotion will increase the tendency of vulnerable groups to adopt the "struggle" strategy.

(2) From the perspective of the vulnerable group, $q$ is affected by both $r_{2}$ and $p$ as shown in Figures 2(a) and 2(b). The analysis process is similar to (1).

\section{Conclusion}

Stakeholders have different understandings and attitudes towards the same incident due to the different ponderances and complexities of social contradictions. When all problems are intertwined and accumulated, both sides of the powerful and vulnerable groups are on the rope and it is difficult for them to find a solution in a rational way. Moreover, special events occur in special times and occasions when it is easier to give rise to a head-on confrontation between the powerful group and the vulnerable group. Thus, the probability of a mass emergency with maintained legal rights increases. Based on the RDEU theory, the author established the RDEU game model for mass emergencies with maintained legal rights and analysed the influence of different emotions on the decision-making behaviour of powerful and vulnerable groups. Results show that both groups tend to choose risky strategies, such as "tough" or "struggle," when both powerful and vulnerable groups hold a "pessimistic emotion"; the probability that they will adopt the risky strategy increases as the degree of "pessimistic emotion" increases. When both the powerful and vulnerable groups hold an "optimistic emotion," they tend to adopt conservative strategies, such as "conservative" or "compromised." The probability that they will adopt the conservative strategy will increase as the degree of "optimistic emotion" increases. Therefore, in mass emergencies with maintained legal rights, the pessimistic emotion will turn the vulnerable group more irrational and the conflict between both sides will grow. Under the influence of emotion, one side of the game will be affected by the strategy that the other party adopts in the game. When the probability that the powerful group adopts a "cooperation" strategy is lower than the critical value, the probability that the vulnerable group adopts the "compromised" strategy will increase as the sentiment index increases. In other words, the vulnerable group expects that when the powerful group does not give in and holds a tough attitude, the vulnerable group will adopt the "compromised" strategy. By contrast, when the probability that the powerful group adopts the "cooperation" strategy is greater than the critical value, the probability that the vulnerable group adopts the "compromised" strategy will decrease as the sentiment index increases. In other words, the vulnerable group expects that the powerful group will work with them. Thus, they will obtain greater benefits if they adopt the "struggle" strategy. Based on the assumptions in this study, the author revealed the law of the influence of emotional factors on decision-making in mass emergencies with maintained legal rights and effectively explained the mechanism of occurrence and evolution of mass emergencies.

By combining the established game model and the simulation results, the occurrence and development of mass emergencies with maintained legal rights can be effectively and reasonably gained from the following points. (1) Improve the violation cost of powerful groups $C_{3}$, promote the openness and transparency of relevant government affairs workflow and compensation standards, formulate strict regulations and systems, and strengthen the supervision and administration of party and government offices. When land acquisition violations occur, forceful advancement or speculations between officials emerge and they should be punished severely. (2) Improve the behavioural costs of powerful groups $C_{1}$, and improve the proportion of social opinions in the decisionmaking of social affairs to ensure that the decision of social affairs of immediate concern to the masses truly reflects the intention of the masses. The openness and transparency of information to strengthen the influence of social opinion 
should be examined to enable the powerful group to bear the loss of reputation due to the assumed surge of illegal behaviour under the great pressure from public opinion. Relevant legal services and consultation and assistance services should be provided to the vulnerable group to raise their awareness of protecting their rights and to expand the correct ways and means of rights protections to further raise the cost that the powerful group suffers in cracking down on the vulnerable group. (3) Enhance the government's credibility and timely monitor public sentiment. Once mass emergencies with maintained legal rights occur, based on the assumptions made in this study, the responsible party must actively investigate to collect evidence of these events. If the government and relevant administrative departments discover that the vulnerable groups have set changes in their emotion, they need to promptly divert the public's bad emotions in case a collective outbreak of negative emotions of the group occurs. The government should provide timely psychological comfort and psychological intervention and avoid the spread of bad emotions to false information, such as rumors.

\section{Data Availability}

The data used to support the findings of this study are available from the corresponding author upon request.

\section{Conflicts of Interest}

The authors declare that they have no conflicts of interest.

\section{Acknowledgments}

This work is supported by the Hebei Provincial Social Science Fund Annual Project (No. HB16GL088): Research on Knowledge Discovery Mechanism and Decision - making Model Based on Wisdom of the collective under "Internet +" Environment.

\section{References}

[1] Y. Jianrong, "Major Types and Basic Characteristics of Group Event in Today's China," Journal of CUPL, vol. 6, pp. 114-120, 2009.

[2] L. Dehai, Evolution and Evaluation of Group Burst Time, Chinese Social Sciences Press, Beijing, China, 2012.

[3] J. von Neumann and O. Morgenstern, Theory of Games and Economic Behaviour, Princeton University Press, 1944.

[4] D. Lee, "Game theory and neural basis of social decision making," Nature Neuroscience, vol. 11, no. 4, pp. 404-409, 2008.

[5] D. Kahneman and A. Tversky, "Prospect theory: an analysis of decision under risk," Econometrica, vol. 47, no. 2, pp. 263-292, 1979.

[6] P. J. Zak and A. Fakhar, "Neuroactive hormones and interpersonal trust: International evidence," Economics \& Human Biology, vol. 4, no. 3, pp. 412-429, 2006.

[7] A. G. Sanfey, J. K. Rilling, J. A. Aronson, L. E. Nystrom, and J. D. Cohen, "The neural basis of economic decision-making in the Ultimatum Game," Science, vol. 300, no. 5626, pp. 1755-1758, 2003.

[8] D. Tankersley, C. J. Stowe, and S. A. Huettel, "Altruism is associated with an increased neural response to agency," Nature Neuroscience, vol. 10, no. 2, pp. 150-151, 2007.

[9] A. G. Sanfey, "Social decision-making: Insights from game theory and neuroscience," Science, vol. 318, no. 5850, pp. 598602, 2007.

[10] J. Quiggin, “A theory of anticipated utility," Journal of Economic Behavior \& Organization, vol. 3, no. 4, pp. 323-343, 1982.

[11] G. Q. Xiong and X. Zhao, "The system dynamics model and evolution simulation on coupling-emotional group events," Systems Engineering, vol. 5, pp. 112-120, 2016.

[12] R Gong and S. O. Business, "Nash equilibrium of hawk-dove game based on rank-dependent expected utility theory," Journal of Management Sciences in China, vol. 15, no. 9, pp. 35-45, 2012.

[13] L. Fang, S. Zhang, T. Zhou et al., "Rank-dependent expected utility model of unexpected incidents considering emotional factor," Journal of University of Electronic Science \&Technology of China, no. 2, pp. 34-40, 2015.

[14] G. Q. Xiong, T. Zhang, and H. T. Wang, "Analysis of the RDEU game model of group conflicts influenced by emotions," Chinese Journal of Management Science, vol. 39, no. 9, pp. 162-170, 2015.

[15] J. F. Chen, R. Chai et al., "Hypergame evolutionary analysis of mass emergency derived from environmental pollution: based on the case of dalian Px event," Systems Engineering, vol. 35, no. 02, pp. 51-59, 2017.

[16] D. H. Liu, X Bao, and X. N. Wang, "How does the pessimistic or optimistic emotion influence the game equilibrium outcome in incidents of violence and terrorism," vol. 25 , no. 10 , pp. 80-88, 2017.

[17] People's Daily Online: “A Conflict between a construction project and villagers in Jinning has caused 8 deaths and 18 injuries", http://yn.people.com.cn/news/yunnan/n/2014/1015/ c228496-22611234.html. 


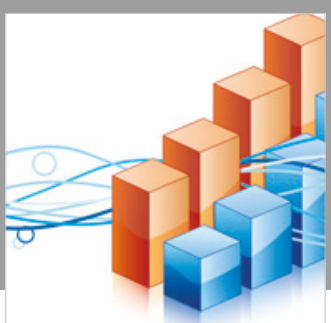

Advances in

Operations Research

\section{-n-m}
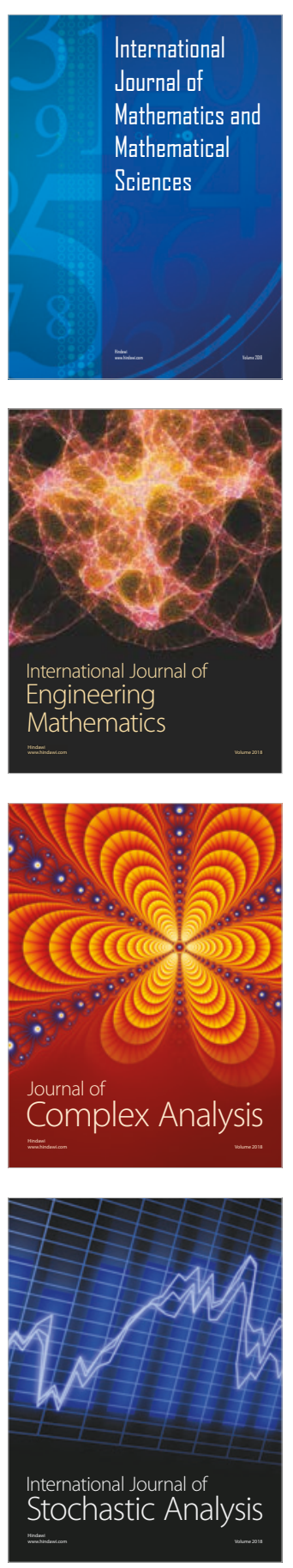
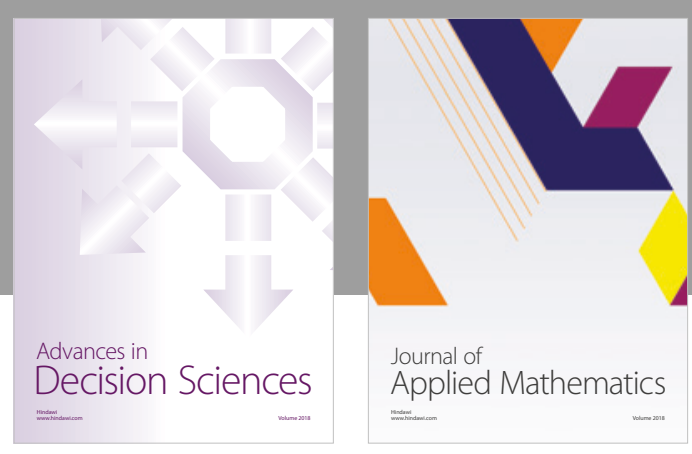

Journal of

Applied Mathematics
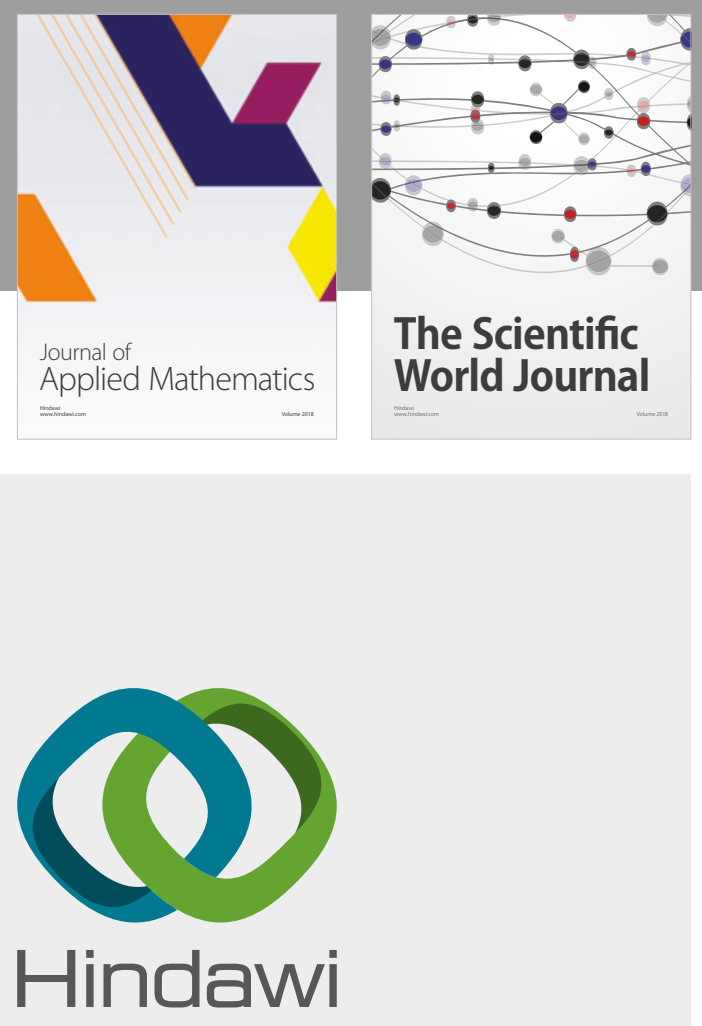

Submit your manuscripts at

www.hindawi.com

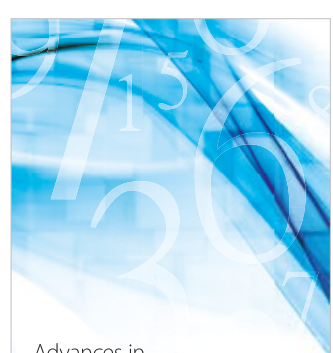

Advances in
Numerical Analysis
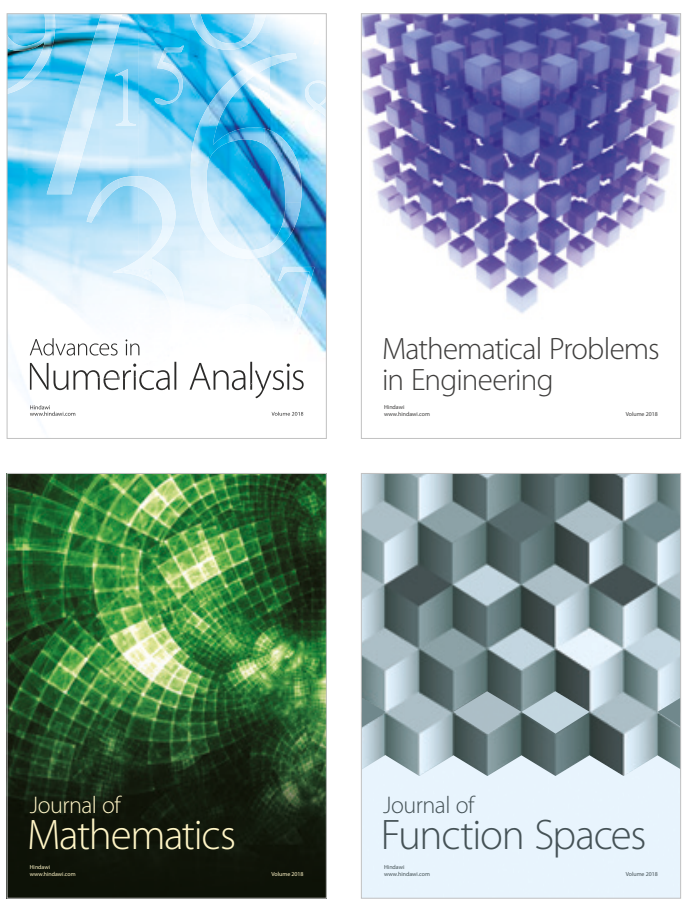

Mathematical Problems in Engineering

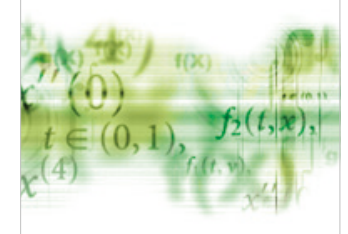

International Journal of

Differential Equations

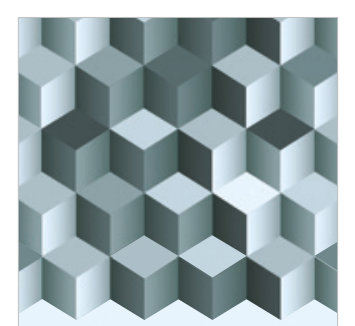

Journal of

Function Spaces

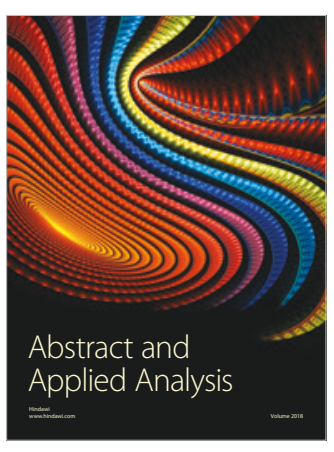

The Scientific

World Journal

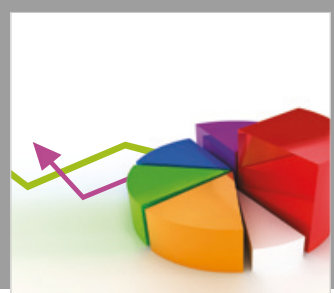

Journal of

Probability and Statistics
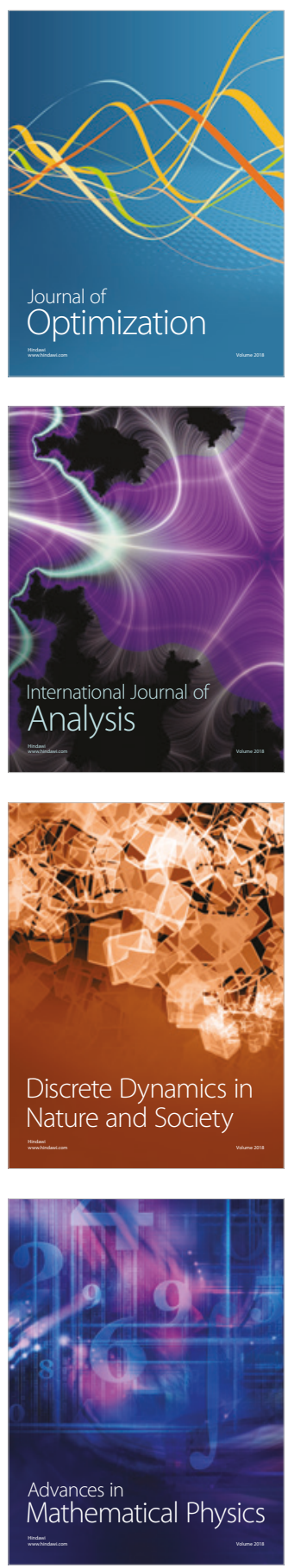